\title{
Collateral cardiovascular damage during the COVID-19 pandemic
}

\author{
Ramesh Nadarajah (1) and Chris P. Gale
}

\begin{abstract}
Care pathways for ST-segment elevation myocardial infarction (STEMI) were interrupted during the coronavirus disease 2019 (COVID-19) pandemic.

A new cardiac MRI study has revealed that increased total ischaemic time for patients with STEMI during major public health restrictions was associated with increased infarct size and other markers of myocardial damage.
\end{abstract}

\begin{abstract}
Refers to Lechner, I. et al. Impact of COVID-19 pandemic restrictions on ST-elevation myocardial infarction: a cardiac magnetic resonance imaging study. Eur. Heart J. https://doi.org/10.1093/eurheartj/ehab621 (2021).
\end{abstract}

The reorganization of health-care services and the implementation of major public health restrictions during the coronavirus disease 2019 (COVID-19) pandemic reduced mortality and morbidity that was directly related to the contagion, but indirectly resulted in 'collateral damage' by interrupting care pathways for diseases other than COVID-19. ST-segment elevation myocardial infarction (STEMI) is a cardiovascular emergency that requires timely reperfusion, typically by means of primary percutaneous coronary intervention (PPCI). Optimal provision of PPCI is dependent on the implementation of 'hub' and 'spoke' networks of hospitals linked by a prioritized and efficient ambulance service $^{1}$. International reports pertaining to admission of patients with STEMI to hospital during the pandemic described increased mortality after PPCI and longer durations between the time of symptom onset and treatment $^{2}$. The MARINA-STEMI cardiac MRI study ${ }^{3}$ is the latest report on the myocardial tissue damage characteristics of patients with STEMI who were admitted to a hospital during the COVID-19 pandemic.

The prospective MARINA-STEMI cohort study, which involves patients presenting with STEMI to a regional PPCI centre in Austria, provides mechanistic insights regarding the effect of major public health restrictions on the extent of myocardial tissue damage in these patients $s^{3}$. In this study, patients who were clinically stable after a STEMI underwent cardiac MRI between three and five days after the myocardial infarction event. The investigators compared 48 patients treated during periods in 2020 when major public health restrictions were implemented with 101 patients treated during periods in 2020 without public health restrictions and 124 patients treated during the corresponding periods between 2015 and 2019 (pre-pandemic). The investigators used cardiac MRI findings to assess infarct size, defined as percentage of the entire left ventricular myocardial mass, as well as the frequency and extent of microvascular obstruction and intramyocardial haemorrhage. Each of these markers of myocardial damage has prognostic relevance: a $5 \%$ increase in infarct size as measured by cardiac MRI contributes to a $19 \%$ increase in all-cause mortality at one year ${ }^{4}$. Furthermore, microvascular obstruction is strongly associated with mortality and hospitalization for heart failure within one year ${ }^{5}$ and intramyocardial haemorrhage is associated with persistent iron in the infarct core, which is predictive of adverse cardiac remodelling and worsening cardiac function ${ }^{6}$.

Notably, baseline patient demographics and comorbidity profiles did not differ significantly between groups, and neither did periprocedural or procedural factors such as pharmacological therapy, frequency of coronary stenting or the frequency of thrombolysis
The scale of disruption to STEMI care pathways was not fully anticipated before the pandemic

in myocardial infarction (TIMI) flow 3 after $\mathrm{PPCI}^{3}$. However, during periods with major public health restrictions, the median total ischaemic time in admitted patients was $75 \mathrm{~min}$ longer and the proportion of patients with TIMI flow 0 before PPCI was 19\% higher than during periods without restrictions ${ }^{3}$. In addition, patients treated during periods of major public health restrictions had more deleterious findings on cardiac MRI than patients in the other groups, including a larger infarct size, more frequent and larger extent of microvascular obstruction, and more frequent intramyocardial haemorrhage.

The relationship between myocardial ischaemic time and adverse myocardial function has been well established for more than 50 years ${ }^{7}$. Although the present study is only observational and causation cannot be inferred, the data add to mounting evidence of the adverse population-level effects associated with delayed times between symptom onset and treatment of STEMI during the COVID-19 pandemic ${ }^{2}$. Moreover, the study investigators are likely to have underestimated the consequences of interruptions of STEMI care pathways during the pandemic because they did not include patients who were positive for COVID-19, patients who were clinically unstable after PPCI and the many individuals with acute MI who died in the community ${ }^{8,9}$.

So what does this study add to our knowledge base? Although there is evidence that public mandates to 'lock down' and the resulting delays in seeking help have resulted in excess deaths, this study describes a potential mechanism by which patients with STEMI were subjected to a higher risk of major adverse cardiovascular events despite receiving guideline-recommended care. This observation highlights the importance of addressing the continuum of care for STEMI, including symptom onset, first medical contact, and ambulance transport and transfer ${ }^{1}$ (FIC. 1). The health-care pathway relies on communication and process flows between multiple teams across pre-hospital and hospital settings and thus was particularly vulnerable to the rapid 
Patient-related delays

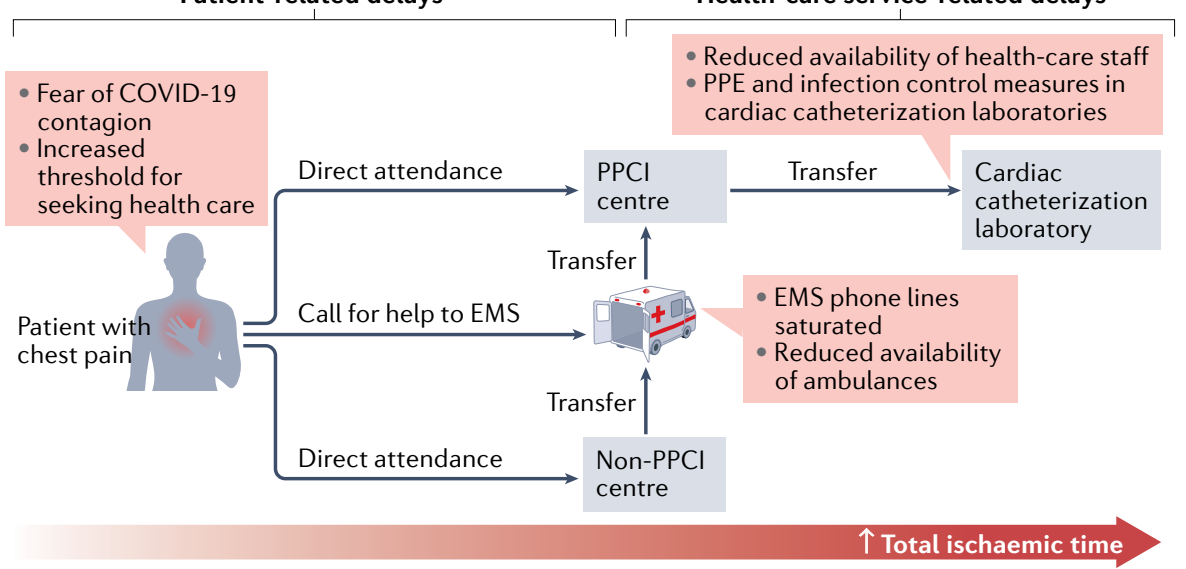

Fig. 1 | Factors that contributed to delayed treatment of STEMI during the COVID-19

pandemic. Numerous patient-related and health-care service-related delays associated with major public health restrictions during the coronavirus disease 2019 (COVID-19) pandemic together prolong total ischaemic time in patients with ST-segment elevation myocardial infarction (STEMI). EMS, emergency medical services; PPE, personal protective equipment; $\mathrm{PPCl}$, primary percutaneous coronary intervention.

reorganization and overload of health-care services during the pandemic.

As we enter a new era of 'living with' COVID-19, research demonstrating possible ways to avoid collateral cardiovascular damage during future waves of infection will have immediate utility. The scale of disruption to STEMI care pathways was not fully anticipated before the pandemic, and might have been exacerbated by policies and communication strategies. During the initial stages of the pandemic, patients feared the potential COVID-19 contagion and did not wish to add additional workload to health-care services that were viewed to be under severe strain. Coordinated regional campaigns from cardiovascular societies, charities and public health institutions to raise awareness of the importance of seeking medical attention for symptoms of MI have resulted in faster normalization of hospital attendance rates for MI to pre-pandemic levels compared to regions where no such campaigns were conducted ${ }^{10}$. Furthermore, pre-hospital networks might need to be fortified, especially in low-income and middle-income countries, to ensure that patients arrive expediently at PPCI centres for treatment. Going forward, health-care services will need to create additional capacity to manage the morbidity that will arise from extra myocardial tissue damage sustained by patients with delayed treatment for STEMI during the pandemic, and plan to protect existing STEMI care pathways in the event of future waves of COVID-19-related infection.
Ramesh Nadarajah (D) ${ }^{1,2}$ and Chris P. Gale ${ }^{1,2}$ 'Leeds Institute for Cardiovascular and Metabolic Medicine, University of Leeds, Leeds, UK.

${ }^{2}$ Department of Cardiology, Leeds Teaching Hospitals NHS Trust, Leeds, UK.

凶e-mail:r.nadarajah@leeds.ac.uk

https://doi.org/10.1038/s41569-021-00661-x

1. Kontos, M. C. et al. Prehospital activation of hospital resources (PreAct) ST-segment-elevation myocardial infarction (STEMI): a standardized approach to prehospital activation and direct to the catheterization laboratory for STEMI recommendations from the American Heart Association's Mission: Lifeline Program. J. Am. Heart Assoc. 9, e011963 (2020).

2. Chew, N. W. et al. The global impact of the COVID-19 pandemic on STEMI care: a systematic review and meta-analysis. Can. J. Cardiol. 37, 1450-1459 (2021).

3. Lechner, I. et al. Impact of COVID-19 pandemic restrictions on ST-elevation myocardial infarction: a cardiac magnetic resonance imaging study. Eur. Heart J. https://doi.org/10.1093/eurheartj/ ehab621 (2021).

4. Stone, G. W. et al. Relationship between infarct size and outcomes following primary PCI: patient-level analysis from 10 randomized trials. J. Am. Coll. Cardiol. 67, 1674-1683 (2016).

5. de Waha, S. et al. Relationship between microvascular obstruction and adverse events following primary percutaneous coronary intervention for ST-segment elevation myocardial infarction: an individual patient data pooled analysis from seven randomized trials. Eur. Heart J. 38, 3502-3510 (2017).

6. Carberry, J et al. Persistent iron within the infarct core after ST-segment elevation myocardial infarction: implications for left ventricular remodeling and health outcomes. JACC Cardiovasc. Imaging 11, 1248-1256 (2018).

7. Maroko, P. R. et al. Factors influencing infarct size following experimental coronary artery occlusions. Circulation 43, 67-82 (1971).

8. Wu, J. et al. Place and underlying cause of death during the COVID-19 pandemic: retrospective cohort study of 3.5 million deaths in England and Wales, 2014 to 2020. Mayo Clin. Proc. 96, 952-963 (2021).

9. Wu, J. et al. Place and causes of acute cardiovascular mortality during the COVID-19 pandemic. Heart 107 113-119 (2021).

10. Van Belle, E. et al. Myocardial infarction incidence during national lockdown in two French provinces unevenly affected by COVID-19 outbreak: an observational study. Lancet Reg. Health Eur. 2, 100030 (2021).

Competing interests

The authors declare no competing interests. 